GANIT J. Bangladesh Math. Soc. (ISSN 1606-3694) 37 (2017) 73-92

\title{
NUMERICAL STUDY OF UNSTEADY FLUID FLOW AND HEAT TRANSFER THROUGH A ROTATING CURVED RECTANGULAR CHANNEL
}

\author{
Md. Zohurul Islam ${ }^{1}$, Md. Arifuzzaman ${ }^{2}$ and Rabindra Nath Mondal $^{3 *}$ \\ 1) Department of Mathematics, Jessore University of Science and Technology, \\ Jessore-7408, Bangladesh \\ ${ }^{2)}$ Department of General Educational Development, Faculty of Science and Information \\ Technology, Daffodil International University, Dhaka, Bangladesh \\ ${ }^{3)}$ Department of Mathematics, Jagannath University, Dhaka-1100, Bangladesh \\ ${ }^{*}$ Corresponding author: rnmondal71@yahoo.com
}

Received 06.02.2017 Accepted 26.10.2017

\begin{abstract}
Numerical study of unsteady fluid flow and heat transfer through a rotating curved rectangular channel with aspect ratio 2 and curvature ratio 0.05 has been performed by using a spectral-based numerical method, and covering a wide range of the rotational parameter, the Taylor number $\mathrm{Ta}$, for both the positive and negative rotation of the channel. In this paper, unsteady flow characteristics are investigated under combined action of the centrifugal, Coriolis and buoyancy forces for the Dean number $D e=1000$. For positive rotation, we investigated unsteady solutions for $0 \leq T a \leq 500$, and it is found that the chaotic flow turns into steady-state flow through periodic or multi-periodic flows. For negative rotation, on the other hand, unsteady solutions are investigated for $-500 \leq T a \leq 0$, and it is found that the unsteady flow undergoes through various flow instabilities. Typical contours of secondary flow patterns and temperature profiles are obtained at several values of $\mathrm{Ta}$, and it is found that the unsteady flow consists of asymmetric two- to eightvortex solutions. The present study shows that convective heat transfer is significantly enhanced as the secondary flow becomes stronger and the chaotic flow enhances heat transfer more effectively than the steady-state or periodic solutions.
\end{abstract}

Keywords: Rotating curved channel; secondary vortex; unsteady solutions; Dean number, Taylor number, time evolution.

\section{Introduction}

Investigation of flow and heat transfer through curved ducts and channels is an interesting subject for researchers in the past and the present. These channels are extensively used in many engineering applications, such as in turbo-machinery, refrigeration, air conditioning systems, heat exchangers, rocket engine, internal combustion engines and blade-to-blade passages in modern gas turbines. In a curved duct, centrifugal forces are developed in the flow due to channel curvature causing a counter rotating vortex motion applied on the axial flow through the channel. This 
creates characteristics spiraling fluid flow in the curved passage known as secondary flow. At a certain critical flow condition and beyond, additional pairs of counter rotating vortices appear on the outer concave wall of the curved fluid passage which are known as Dean vortices, in recognition of the pioneering work in this field by Dean [1]. After that, many theoretical and experimental investigations have been done by keeping this flow in mind; for instance, the articles by Berger et al. [2], Nandakumar and Masliyah [3] and Ito [4] may be referenced.

The fluid flowing in a rotating curved duct is subjected to two forces: the Coriolis force due to rotation and the centrifugal force due to curvature. Such rotating passages are used in cooling system for conductors of electrical generators. For isothermal flows of a constant property fluid, the Coriolis force tends to generate vortices while centrifugal force is purely hydrostatic (Zhang et al. [5]). When a temperature induced variation of fluid density occurs for non-isothermal flows, both Coriolis and centrifugal type buoyancy forces can contribute to the formation of vortices. These two effects of rotation either enhance or counteract each other in a non-linear manner depending on the direction of wall heat flux and the flow domain. Therefore, the effect of system rotation is more subtle and complicated, and yields new richer features of flow and heat transfer for the non-isothermal flows. Ishigaki [6] examined the flow structure and friction factor numerically for both the counter-rotating and co-rotating curved circular pipe with small curvature. Selmi et al. [7] and Dennis and $\mathrm{Ng}$ [8] examined combined effects of system rotation and curvature on the bifurcation structure of two-dimensional flows in a rotating curved duct with square cross section. Miyazaki [9] examined the solution when the rotation is in the same direction as the Coriolis force emphasizing the centrifugal force caused by the duct curvature, which is known as co-rotating case. Selmi and Nandakumer [10] performed numerical studies on the flow characteristics in rotating curved rectangular ducts. Wang and Cheng $[11,12]$ and Daskopoulos and Lenhoff, [13] carried out a bifurcation study of the flow through a circular pipe and employed finite volume method. They examined the flow characteristics and heat transfer in curved square ducts for positive rotation and found reverse secondary flow for the co-rotation cases.

Time-dependent analysis of fully developed curved duct flows was initiated by Yanase and Nishiyama [14] for a rectangular cross section. In that study, they investigated unsteady solutions for the case where dual solutions exist. The unsteady behavior of the flow in a curved rectangular duct of large aspect ratio was investigated, in detail, by Yanase et al. [15] numerically. They performed timeevolution calculations of the unsteady solutions with and without symmetry condition. Wang and Yang [16] performed numerical as well as experimental investigations of periodic oscillations for fully developed flow in a curved square duct. They showed, both experimentally and numerically, that a temporal oscillation takes place between symmetric/asymmetric 2-cell and 4-cell flows when there are no stable steady solutions. Mondal et al. [17] performed numerical prediction of non-isothermal flows through a rotating curved square duct and revealed some of such new features. Very recently, Mondal et al. $[18,19]$ investigated combined effects of centrifugal and Coriolis instability of the isothermal/non- 
isothermal flows through a rotating curved rectangular duct numerically. The secondary flow characteristics in a curved square duct were investigated experimentally by using visualization method by Yamamoto et al. [20]. Three-dimensional incompressible viscous flow and heat transfer in a rotating U-shaped square duct were studied numerically by Nobari et al. [21]. However, transient behavior of the unsteady solutions is not yet resolved, in detail, for the flow through a rotating curved rectangular duct of small curvature in the case of strong centrifugal force, which motivated the present study to fill up this gap.

One of the most important applications of curved duct flow is to enhance the thermal exchange between two sidewalls, because it is possible that the secondary flow may convey heat and then increases heat flux between two sidewalls. Chandratilleke and Nursubyakto [22] presented numerical calculations to describe secondary flow characteristics in the flow through curved ducts of aspect ratios ranging from 1 to 8 that were heated on the outer wall, where they studied for small Dean numbers and compared the numerical results with their experimental data. Yanase et al. [23] and Mondal et al. [24] studied time-dependent behavior of the unsteady solutions for curved rectangular/square duct flow and showed that secondary flows enhance heat transfer in the flow. Recently Norouzi et al. [25] investigated fully developed flow and heat transfer of viscoelastic materials in curved square ducts under constant heat flux. To the best of the authors' knowledge, however, there has not yet been done any substantial work studying the effects of rotation on the unsteady flow behavior for the non-isothermal flow through a rotating curved rectangular duct in the presence of buoyancy force with large pressure gradient.

In this paper, we investigate time-dependent flow behavior through a loosely coiled rotating rectangular duct by using a spectral-based numerical scheme, and show an enhancement of convective heat transfer by secondary flows. Studying the effects of rotation on the unsteady flow characteristics, caused by the combined action of centrifugal, Coriolis and buoyancy forces, is an important objective of the present study.

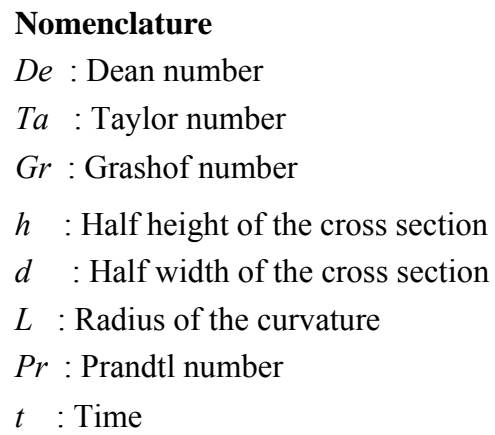




\section{Flow Model and Governing Equations}

Consider a hydro-dynamically and thermally fully developed two-dimensional flow of viscous incompressible fluid through a rotating curved duct with rectangular cross section, whose height and width are $2 h$ and $2 d$, respectively. The coordinate system with relevant notation is shown in Fig. 1, where $x^{\prime}$ and $y^{\prime}$ axes are taken to be in the horizontal and vertical directions respectively, and $z^{\prime}$ is the axial direction. The system rotates at a constant angular velocity $\Omega$ around the $y^{\prime}$ axis. It is assumed that the outer wall of the duct is heated while the inner wall cooled. It is also assumed that the flow is uniform in the axial direction, which is driven by a constant pressure gradient $G$ along the center-line of the duct.

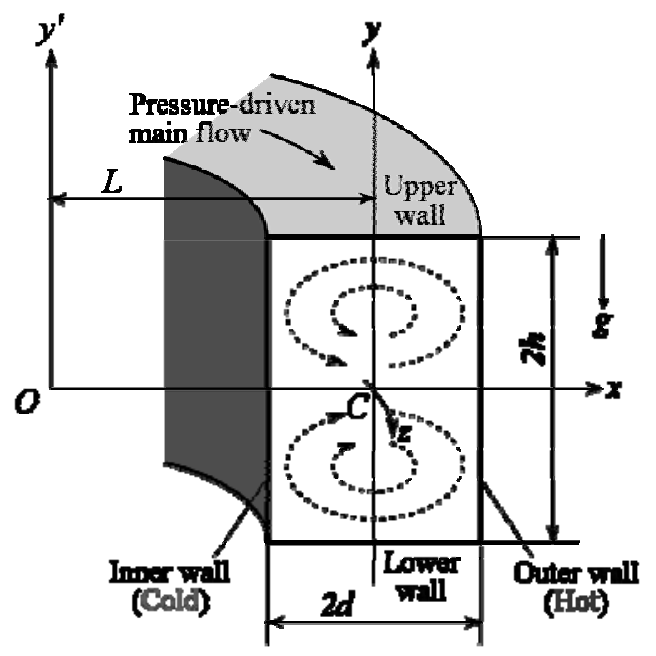

Figure 1. Coordinate system of the curved duct

The continuity, Navier-Stokes and energy equations, in terms of dimensional variables, are expressed as:

Continuity equation: $\frac{\partial u^{\prime}}{\partial r^{\prime}}+\frac{\partial v^{\prime}}{\partial y^{\prime}}+\frac{u^{\prime}}{r^{\prime}}=0$,

Momentum equations:

$$
\begin{aligned}
& \frac{\partial u^{\prime}}{\partial t^{\prime}}+u^{\prime} \frac{\partial u^{\prime}}{\partial r^{\prime}}+v^{\prime} \frac{\partial u^{\prime}}{\partial y^{\prime}}-\frac{w^{\prime 2}}{r^{\prime}}=-\frac{1}{\rho} \frac{\partial P^{\prime}}{\partial r^{\prime}}+\mathrm{v}\left[\frac{\partial^{2} u^{\prime}}{\partial r^{\prime 2}}+\frac{\partial^{2} u^{\prime}}{\partial y^{\prime 2}}+\frac{1}{r^{\prime}} \frac{\partial u^{\prime}}{\partial r^{\prime}}-\frac{u^{\prime}}{r^{\prime 2}}\right] \\
& \frac{\partial v^{\prime}}{\partial t^{\prime}}+u^{\prime} \frac{\partial v^{\prime}}{\partial r^{\prime}}+v^{\prime} \frac{\partial v^{\prime}}{\partial y^{\prime}}=-\frac{1}{\rho} \frac{\partial P^{\prime}}{\partial y^{\prime}}+v\left[\frac{\partial^{2} v^{\prime}}{\partial r^{\prime 2}}+\frac{1}{r^{\prime}} \frac{\partial v^{\prime}}{\partial r^{\prime}}-\frac{\partial^{2} v^{\prime}}{\partial y^{\prime 2}}\right]+g \beta T^{\prime}
\end{aligned}
$$




$$
\frac{\partial w^{\prime}}{\partial t^{\prime}}+u^{\prime} \frac{\partial w^{\prime}}{\partial r^{\prime}}+v^{\prime} \frac{\partial w^{\prime}}{\partial y^{\prime}}+\frac{u^{\prime} w^{\prime}}{r^{\prime}}=-\frac{1}{\rho} \frac{1}{r^{\prime}} \frac{\partial P^{\prime}}{\partial z^{\prime}}+v\left[\frac{\partial^{2} w^{\prime}}{\partial r^{\prime 2}}+\frac{\partial^{2} w^{\prime}}{\partial y^{\prime 2}}+\frac{1}{r^{\prime}} \frac{\partial w^{\prime}}{\partial r^{\prime}}-\frac{w^{\prime}}{r^{\prime 2}}\right]
$$

Energy equation: $\frac{\partial T^{\prime}}{\partial t^{\prime}}+u^{\prime} \frac{\partial T^{\prime}}{\partial r^{\prime}}+v^{\prime} \frac{\partial T^{\prime}}{\partial y^{\prime}}=\kappa\left[\frac{\partial^{2} T^{\prime}}{\partial r^{\prime 2}}+\frac{1}{r^{\prime}} \frac{\partial T^{\prime}}{\partial r^{\prime}}+\frac{\partial^{2} T^{\prime}}{\partial y^{\prime 2}}\right]$,

where $r^{\prime}=L+x^{\prime}$ and $u^{\prime}, v^{\prime}$ and $w^{\prime}$ are the dimensional velocity components in the $x^{\prime}, y^{\prime}$ and $z^{\prime}$ directions respectively and these velocities are zero at the wall. Here, $P^{\prime}$ is the dimensional pressure, $T^{\prime}$ is the dimensional temperature and $t^{\prime}$ is the dimensional time. In the above formulations $\rho, v, \beta, \kappa$ and $g$ are the density, the kinematic viscosity, the coefficient of thermal expansion, the coefficient of thermal diffusivity and the gravitational acceleration, respectively. Thus in Eqs. (1) to (5) the variables with prime denotes the dimensional quantities. The dimensional variables are then non-dimensionalized by using the representative length $d$ and the representative velocity $U_{0}=\mathrm{v} / d$. We introduce the non-dimensional variables defined as:

$$
\begin{aligned}
& u=\frac{u^{\prime}}{U_{0}}, v=\frac{v^{\prime}}{U_{0}}, w=\frac{\sqrt{2 \delta}}{U_{0}} w^{\prime}, x=\left(\frac{x^{\prime}}{d}-\frac{1}{\delta}\right), \bar{y}=\frac{y^{\prime}}{d}, z=\frac{z^{\prime}}{d} \\
& T=\frac{T^{\prime}}{\Delta T^{\prime}}, t=\frac{U_{0}}{d} t^{\prime}, \delta=\frac{d}{L}, P=\frac{P^{\prime}}{\rho U_{0}^{2}}
\end{aligned}
$$

where $u, v$ and $w$ are the non-dimensional velocity component in the $x, \bar{y}$ and $z$ directions, respectively; $t$ is the non-dimensional time, $P$ is the non-dimensional pressure, $\delta$ is the nondimensional curvature and temperature is non-dimensionalized by $\Delta T$. Henceforth, all the variables are non-dimensionalized if not specified.

A new coordinate variable $y$ is then introduced in the $\bar{y}$ direction as $\bar{y}=a y$, where $a=(h / d) \approx 2$ is the aspect ratio of the duct cross section. From now on, y denotes $\bar{y}$ for the sack of simplicity.

Since the flow field is assumed to be uniform in the $z$-direction, the sectional stream function $\psi$ is introduced in the $x$ and $y$ directions as

$$
u=\frac{1}{1+\delta x} \frac{\partial \psi}{\partial y}, \quad v=-\frac{1}{1+\delta x} \frac{\partial \psi}{\partial x}
$$

Then the basic equations for the axial velocity $w$, the stream function $\psi$ and the temperature $T$ are derived from the Navier-Stokes equations and the energy equation as,

$$
\begin{aligned}
(1+\partial x) \frac{\partial w}{\partial t}= & D e-\frac{1}{2} \frac{\partial(w, \psi)}{\partial(x, y)}-\frac{\delta^{2} w}{1+\delta x}+(1+\delta x) \Delta_{2} w-\frac{1}{2} \frac{\delta}{2(1+\delta x)} \frac{\partial \psi}{\partial y} w+\delta \frac{\partial w}{\partial x}-\delta \operatorname{Tr} \frac{\partial \psi}{\partial y} \\
& \left(\Delta_{2}-\frac{\delta}{1+\delta x} \frac{\partial}{\partial x}\right) \frac{\partial \psi}{\partial t}=-\frac{1}{2} \frac{1}{(1+\delta x)} \frac{\partial\left(\Delta_{2} \psi, \psi\right)}{\partial(x, y)}+\frac{1}{2} \frac{\delta}{(1+\delta x)^{2}} \times
\end{aligned}
$$




$$
\begin{aligned}
& {\left[\frac{\partial \psi}{\partial y}\left(2 \Delta_{2} \psi-\frac{3 \delta}{1+\delta x} \frac{\partial \psi}{\partial x}+\frac{\partial^{2} \psi}{\partial x^{2}}\right)-\frac{\partial \psi}{\partial x} \frac{\partial 2 \psi}{\partial x \partial y}\right]+\frac{\delta}{(1+\delta x)^{2}} \times\left[3 \delta \frac{\delta^{2} \psi}{\partial x^{2}}-\frac{3 \delta^{2}}{1+\delta x} \frac{\partial \psi}{\partial x}\right]} \\
& -\frac{2 \delta}{1+\delta x} \frac{\partial}{\partial x} \Delta^{2} \psi+\frac{1}{2} w \frac{\partial w}{\partial y}+\Delta_{2}^{2} \psi-G r(1+\delta x) \frac{\partial T}{\partial x}-\frac{1}{2} T a \frac{\partial \psi}{\partial y} \\
& \frac{\partial T}{\partial t}+\frac{1}{(1+\delta x)} \frac{\partial(T, \psi)}{\partial(x, y)}=\frac{1}{\operatorname{Pr}}\left(\Delta 2 T+\frac{\delta}{1+\delta x} \frac{\partial T}{\partial x}\right)
\end{aligned}
$$

where, $\Delta_{2} \equiv \frac{\partial^{2}}{\partial x^{2}}+\frac{\partial^{2}}{\partial y^{2}}$ and $\frac{\partial(h, k)}{\partial(x, y)} \equiv \frac{\partial h}{\partial x} \frac{\partial k}{\partial y}-\frac{\partial h}{\partial y} \frac{\partial k}{\partial x}$.

In the above formulations, we have used four non-dimensional parameters; $D e$, the Dean number, $T a$, the Taylor number, $G r$, the Grashof number and Pr, the Prandtl number, which are defined as:

$$
D e=\frac{G d^{3}}{\mu v} \sqrt{\frac{2 d}{L}}, T a=\frac{2 \sqrt{2 \delta \Omega_{T} d^{3}}}{v \delta}, G r=\frac{\beta g \Delta T d^{3}}{v^{2}}, \operatorname{Pr}=\frac{v}{\kappa}
$$

The rigid boundary conditions for the axial velocity $(w)$ and the stream function $(\psi)$ are used as

$$
w( \pm 1, y)=w(x, \pm 1)=\psi( \pm 1, y)=\psi(x, \pm 1)=\frac{\partial \psi}{\partial x}( \pm 1, y)=\frac{\partial \psi}{\partial t}(x, \pm 1)=0
$$

and temperature $T$ is assumed to be constant on the walls as

$T(1, y)=1, T(-1, y)=-1, T(x, \pm 1)=x$.

Note that, Eqs. (7) - (9) are invariant under the transformation of the variables

$$
\left.\begin{array}{l}
y \Rightarrow-y \\
w(x, y, t) \Rightarrow w(x,-y, t) \\
\psi(x, y, t) \Rightarrow-\psi(x,-y, t) \\
T(x, y, t) \Rightarrow-T(x,-y, t)
\end{array}\right\}
$$

If the solution satisfies condition (13) it is called a symmetric solution, and if it does not an asymmetric solution. In the present study, only $T a$ varies while $D e, G r, \delta$ and $\operatorname{Pr}$ are fixed as $D e=$ $1000, G r=500, \delta=0.05$ and $\operatorname{Pr}=7.0$ (water).

\section{Numerical Calculations}

\subsection{Method of numerical calculation}

Equations (7) to (9) are solved numerically by using the spectral method. By this method the expansion functions $\phi_{n}(x)$ and $\psi_{n}(x)$ are expressed as

$$
\left.\begin{array}{l}
\phi_{n}(x)=\left(1-x^{2}\right) C_{n}(x), \\
\left.\psi_{n}(x)=1-x^{2}\right)^{2} C_{n}(x)
\end{array}\right\}
$$


where $C_{n}(x)=\cos \left(n \cos ^{-1}(x)\right.$ is the $n$-th order Chebyshev polynomial. $w(x, y, t), \psi(x, y, t)$ and $T(x, y$, $t)$ are expanded in terms of $\phi_{n}(x)$ and $\psi_{n}(x)$ as

$$
\left.\begin{array}{l}
w(x, y, z)=\sum_{m=0}^{M} \sum_{n=0}^{N} w_{m n}(t) \Phi_{m}(x) \Phi_{n}(y), \\
\psi(x, y, t)=\sum_{m=0}^{M} \sum_{n=0}^{N} \Psi_{m n}(t) \Psi_{m}(x) \Psi_{n}(y), \\
T(x, y, t)=\sum_{m=0}^{M} \sum_{n=0}^{N} T_{m n}(t) \Phi_{m}(x) \Phi_{n}(y)+x,
\end{array}\right\}
$$

where $M$ and $N$ are the truncation numbers in the $x$ and $y$ directions respectively. The expansion coefficients $w_{m n}, \psi_{m n}$ and $T_{m n}$ are then substituted into the basic Eqs. (7) - (9) and the collocation method is applied. As a result, the nonlinear algebraic equations for $w_{m n}, \psi_{m n}$ and $T_{m n}$ are obtained. The collocation points are taken to be

$$
x_{i}=\cos \left[\pi\left(1-\frac{i}{M+2}\right)\right], \quad y_{i}=\cos \left[\pi\left(\pi \frac{i}{N+2}\right)\right]
$$

where $i=1, \ldots, M+1$ and $j=1, \ldots, N+1$. Finally, in order to calculate the unsteady solutions, the Crank-Nicolson and Adams-Bashforth methods together with the function expansion (9) and the collocation methods (Gottlieb and Orszag [26]) are applied to Eqs. (7) to (9). Details of the method are available in Mondal et al. [27].

\subsection{The Nusselt Number}

As an index of horizontal heat transfer, the Nusselt number, $N u$, is defined as

$$
N u=-\frac{d^{*}}{\Delta T^{*}}\left\langle\left.\frac{\partial T^{*}}{\partial x^{*}}\right|_{x=0}\right\rangle \text {. }
$$

where the quantities with asterisk $(*)$ denote dimensional ones, $\langle$.$\rangle denotes the mean over the heat$ transfer from the walls to the fluid, $x$ is the distance from the wall, and $\Delta T$ is the temperature difference between the vertical sidewalls. Since the temperature distribution from the walls to the fluid is not uniform due to differentially heated vertical sidewalls, $N u$ is different on both the cooled and heated sidewalls. In this study, for the unsteady solutions, we define the Nusselt number for the cooled $\left(N u_{c}\right)$ and heated $\left(N u_{h}\right)$ sidewalls as follows:

$$
N u_{\tau_{c}}=\frac{1}{2} \int_{-1}^{1}\left\langle\left\langle\left.\frac{\partial T}{\partial x}\right|_{x=-1}\right\rangle\right) d y, n u_{\tau_{h}}=\frac{1}{2} \int_{-1}^{1}\left\langle\left\langle\left.\frac{\partial T}{\partial x}\right|_{x=1}\right\rangle\right) d y
$$

where $\langle\langle\rangle\rangle$ denotes an average over a time interval $\tau$. When the flow field is periodic, $\tau$ is normally taken as one period, and if it is chaotic, $\tau$ is chosen as an appropriate time interval. 


\subsection{Numerical Accuracy}

The accuracy of the numerical calculations is investigated for the truncation numbers $M$ and $N$ used in this study. For good accuracy of the solutions, $N$ is chosen equal to $2 M$. The grid sizes are taken as $12 \times 24,14 \times 28,16 \times 32,18 \times 36$ and $20 \times 40$ as shown in Table 1 , where $\lambda$ is the resistance coefficient and $w(0,0)$ is the axial velocity of the steady solution at $(x, y)=(0,0)$ for $D e=1000, T a=500, G r=500$ and $\delta=0.05$ for the aspect ratio 2. Table 1 shows that $M=16$ and $N=32$ gives sufficient accuracy of the present numerical solutions.

Table 1. The values of $\lambda$ and $w(0,0)$ for various values of $M$ and $N$ for $D e=1000, T a=500, G r=500$ and curvature $\delta=0.05$.

\begin{tabular}{llll}
$M$ & $N$ & $\lambda$ & $w(0,0)$ \\
12 & 24 & 0.16146237 & 747.250 \\
14 & 28 & 0.17762868 & 748.095 \\
$\mathbf{1 6}$ & $\mathbf{3 2}$ & $\mathbf{0 . 1 7 8 4 2 7 9 9}$ & $\mathbf{7 5 4 . 5 5 1}$ \\
18 & 36 & 0.17974814 & 755.866 \\
20 & 40 & 0.17792901 & 749.861 \\
\hline
\end{tabular}

3.4 Resistance coefficient

We use the resistance coefficient $\lambda$ as one of the representative quantities of the flow state. It is also called the hydraulic resistance coefficient, and is generally used in fluids engineering, defined as

$$
\frac{P_{1}^{*}-P_{2}^{*}}{\Delta z^{*}}=\frac{\lambda}{d_{h}^{*}} \frac{1}{2} \rho\left\langle w^{*}\right\rangle^{2},
$$

where quantities with an asterisk denote the dimensional ones, \langle\rangle stands for the mean over the cross section of the duct and $d_{h}^{*}$ is the hydraulic diameter. Since $\left(P_{1}^{*}-P_{2}^{*}\right) / \Delta z^{*}=G, \lambda$ is related to the mean non-dimensional axial velocity $\langle w\rangle$ as

$$
\lambda=\frac{16 \sqrt{2 \delta} D n}{3\langle w\rangle^{2}},
$$

In this paper, $\lambda$ is used to calculate the unsteady solutions by numerical computations.

\section{Results and Discussion}

4.1 Case I: Positive rotation $(0 \leq T r \leq 500)$

In order to study the nonlinear behavior of the unsteady solutions, time evaluation calculations are performed for $-500 \leq T a \leq 500, D e=1000, G r=500$ and curvature $\delta=0.05$. First, we calculate unsteady solutions for positive rotation of the duct $(0 \leq \operatorname{Tr} \leq 500)$. Figure 2 (a) shows time 
evolution of $\lambda$ for $T a=0$. It is found that the unsteady flow at $T a=0$ oscillates irregularly that means the flow is chaotic. This chaotic oscillation is well justified by drawing the phase space of the time evolution result as shown in Fig. 2(b) in the $\gamma-\lambda$ plane, where $\gamma=\iint \psi d x d y$. As seen in Fig. 2(b), the orbit of the flow evolution creates multiple paths, which shows that the flow is chaotic. Figure 2(c) shows streamlines and isotherms for $T a=0$, where it is observed that the streamlines of the secondary flow consist of two opposite vortices; one is an outward flow shown by solid lines and the other one inward flow shown by dotted lines. The right-hand side of each duct box of $\psi$ and $T$ indicates outside direction of the duct. As seen in Fig. 2(c), the unsteady flow for $T a=0$ is an asymmetric two- and four-vortex flow pattern. Figure 3(a) shows time evolution of the unsteady solutions for $T a=80$, where we see that the unsteady flow is also chaotic, and the chaotic behavior is well justified by drawing a phase space of the time evolution solution as shown in Fig 3(b). Figure 3(c) shows typical contours of secondary flow patterns (streamlines) and temperature profiles (isotherms) for $T a=80$, where we see that the unsteady flow at $T a=80$ is an asymmetric two- and four-vortex flow pattern. Then we perform time evolution of $\lambda$ for $T a=100$ as shown in Fig. 4(a). It is found the unsteady flow oscillates in an irregular pattern with different modes of frequency, which shows that the flow is a transitional chaos (Mondal et al. [27]). In order to well identify the nature of the flow oscillation, a phase space of the time evolution result is shown in Fig. 4(b) for $T a=100$, which shows that the flow is a transitional chaos that turns into multi periodic or periodic flows as $T a$ is increased. Typical contours of streamlines and isotherms are then obtained and plotted in Fig. 4(c), where we see that the unsteady flow at $T a=100$ is an asymmetric two-vortex flow pattern.

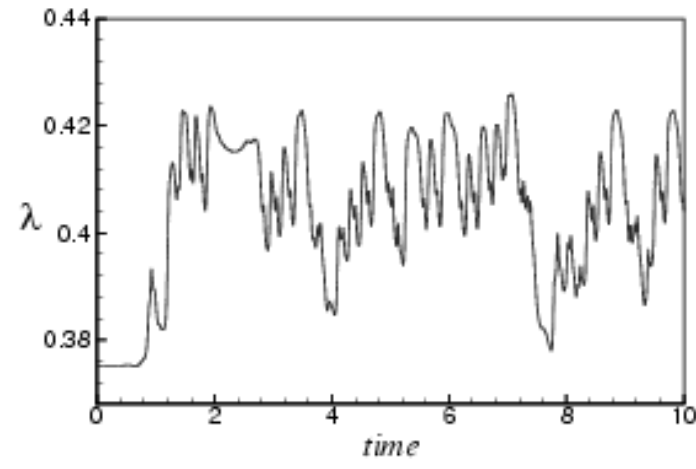

(a)

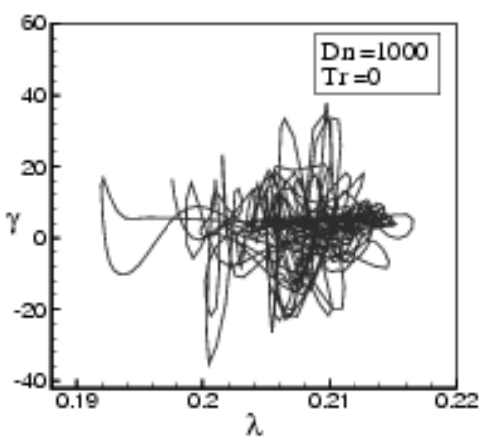

(b)
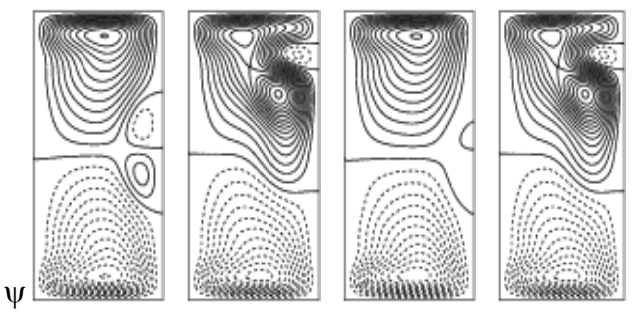

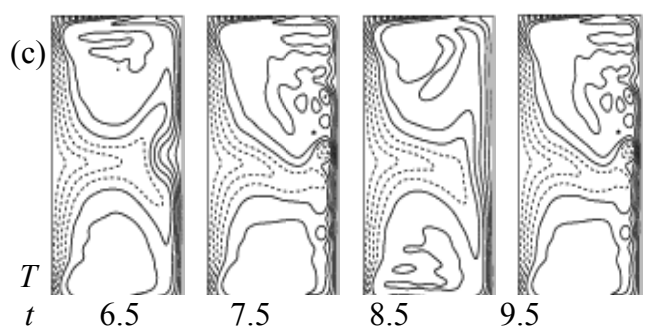

Figure 2. Unsteady results for $T a=0$. (a) Time evolution of $\lambda$, (b) Phase space, (c) Streamlines (top) and isotherms (bottom) at $6.5 \leq t \leq 9.5$

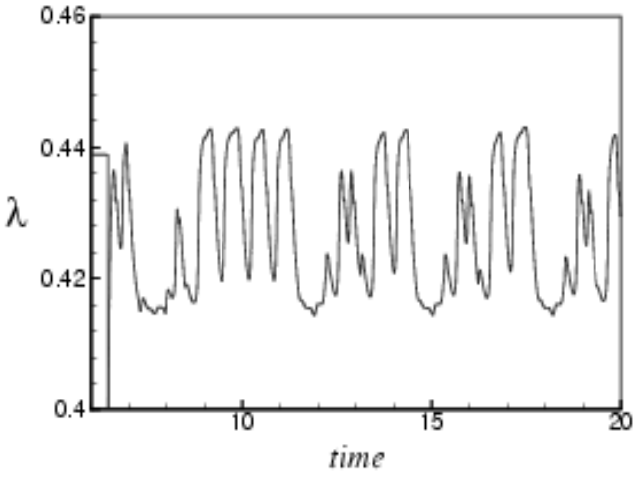

(a)
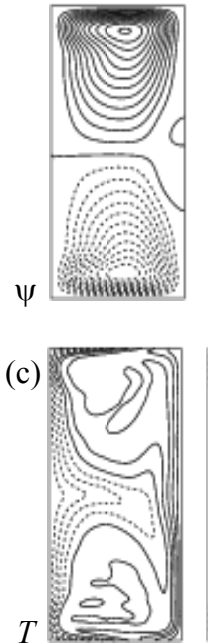

$t \quad 17.00$
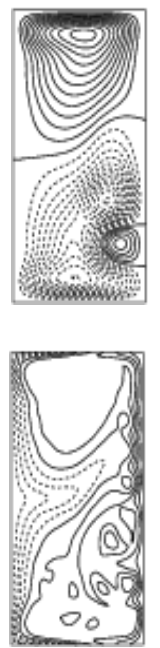

17.50
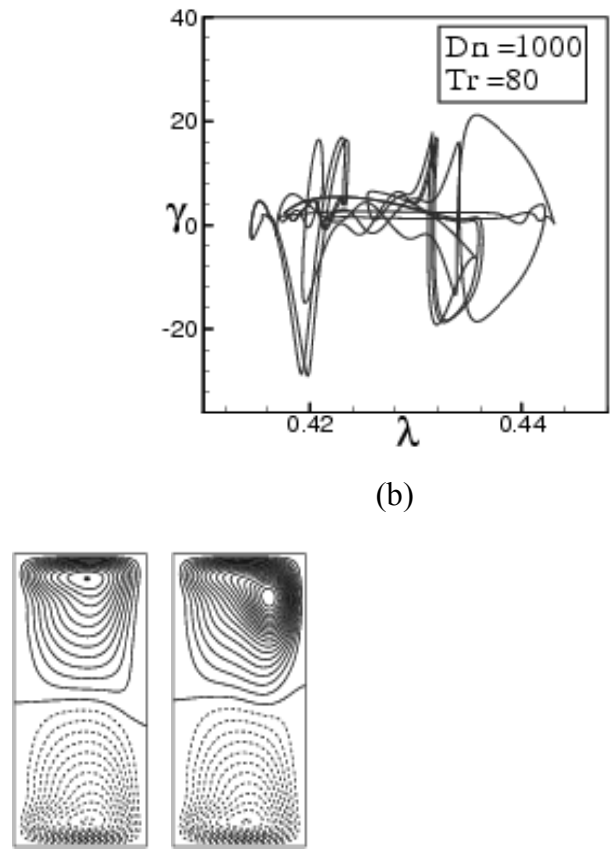

(b)
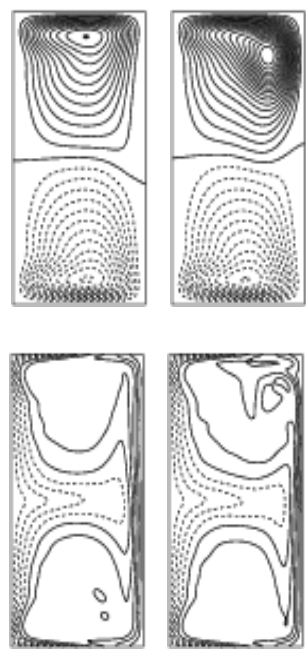

$18.00 \quad 18.50$

Figure 3. Unsteady results for $T a=80$. (a) Time evolution of $\lambda$, (b) Phase space, (c) Streamlines (top) and isotherms (bottom) for $17.0 \leq t \leq 18.5$ 


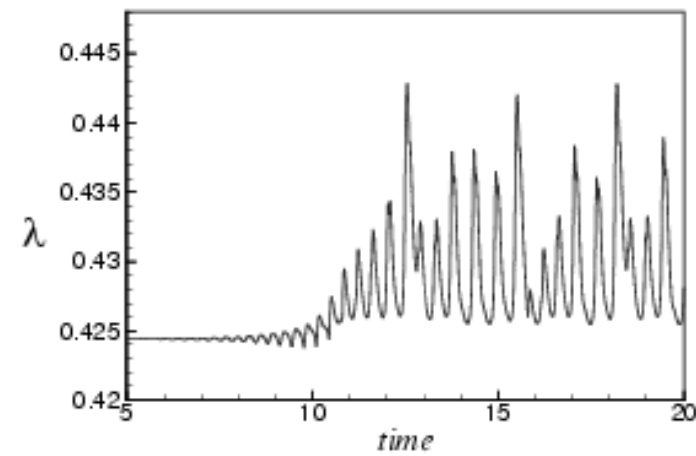

(a)

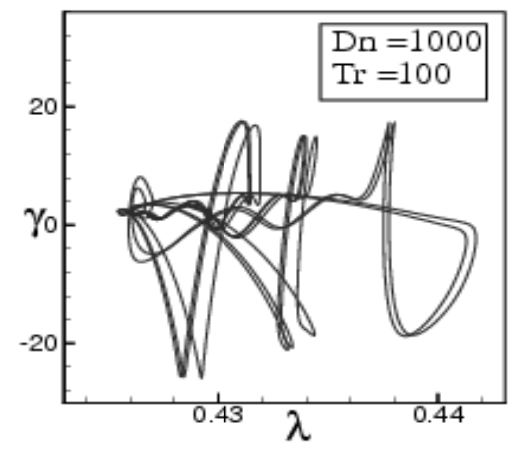

(b)

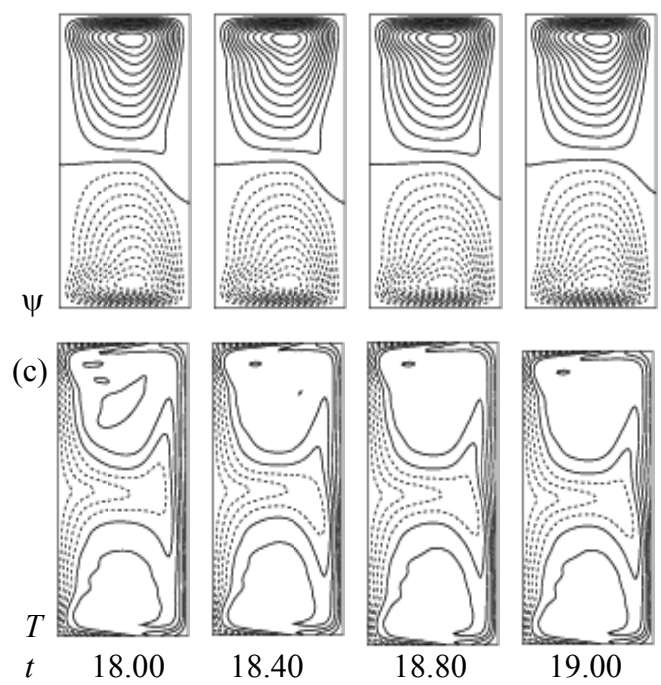

Figure 4. Unsteady results for $T a=100$. (a) Time evolution of $\lambda$, (b) Phase space, (c) Streamlines (top) and isotherms (bottom) for $18.0 \leq t \leq 19.0$

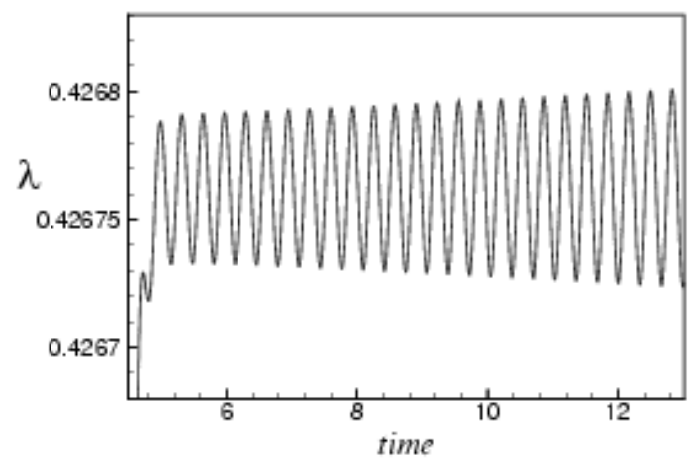

(a)

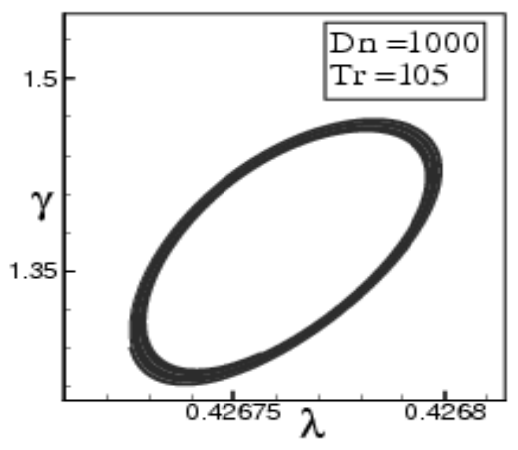

(b) 


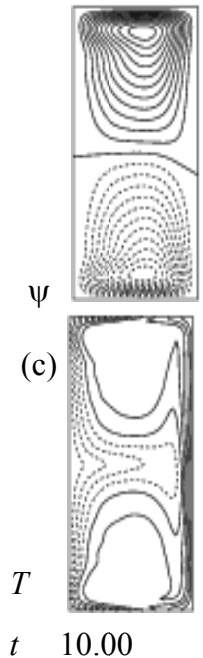

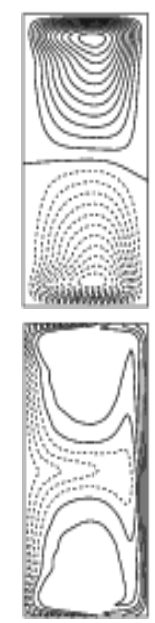

10.40

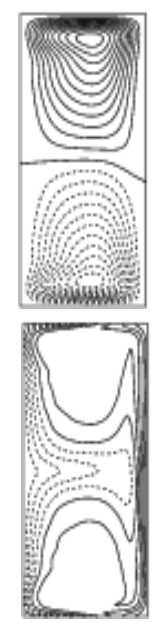

10.80
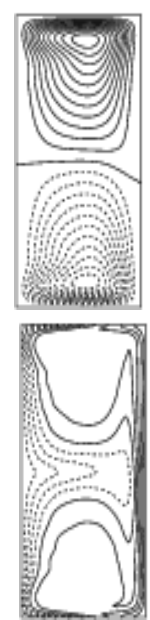

11.00

Figure 5. Unsteady results for $T a=105$. (a) Time evolution of $\lambda$, (b) Phase space, (c) Streamlines (top) and isotherms (bottom) for $10.5 \leq \mathrm{t} \leq 11.0$

Then we performed time evolution of $\lambda$ for $T a=105$ at $D n=1000$ as shown in Fig. 5(a). Figure 5(a) shows that the unsteady flow is a periodic oscillation for $T a=105$, which is well justified by drawing the phase space of the time evolution result as shown in Fig. 5(b). Figure 5(b) shows that the orbit of the oscillation is same at a certain time interval that assures that the fluid particles oscillate periodically. Streamlines and isotherms are shown in Fig. 5(c) for $T a=105$, where we observe that the stream lines are symmetrically distributed and produce asymmetric two-vortex flow pattern. It is also observed that the stream lines of the temperature profiles are found to be consistence with the secondary vortices. Then we increase the rotational speed for $105<T a \leq 500$ and it is found that the unsteady flow is a steady-state solution for any value of $T r$ in this range. Figures 6(a) and 7(a) show, for example, time evolution results for $T a=130$ and $T a=500$ respectively, where steady-state solutions are observed. Since the flow is steady-state, a single contour of each of the secondary flow patterns and temperature profiles is shown in Figs. 6(b) and 7(b) for $T a=130$ and $T a=500$ respectively, where we see that steady-state flow is an asymmetric two-vortex flow pattern.

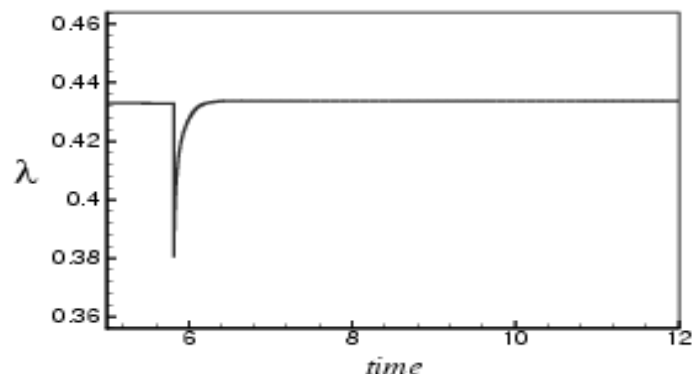

(a)

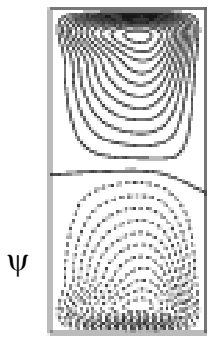

$T$

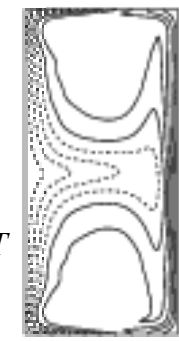

(b)

Figure 6. Unsteady results for $T a=130$. (a) Time evolution of $\lambda$, (b) Streamlines (top) and isotherms (bottom) for $T a=130$ at $t=10$. 
(a)

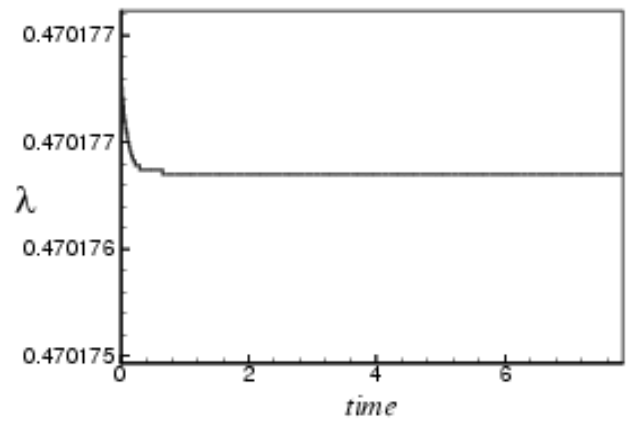

(b)

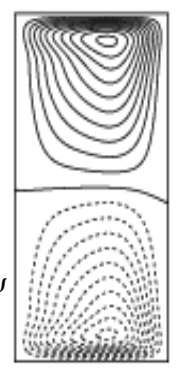

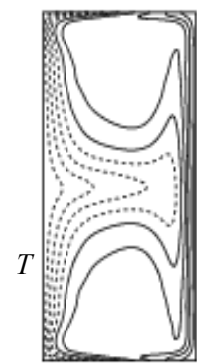

Figure 7. Unsteady results for $T a=500$. (a) Time evolution of $\lambda$, (b) Streamlines (top) and isotherms (bottom) for $T a=500$ at $t=6.0$.

4.2 Case II: Negative rotation $(-500 \leq T a<0)$

We investigated unsteady solutions for negative rotation of the duct at $-500 \leq T a<0$ for the constant pressure gradient $D e=1000$ at curvature $\delta=0.05$. Negative rotation means that the rotational direction is opposite to the main flow direction. Figure 8(a) shows time evolution of $\lambda$ for $T a=-50$ at $D e=1000$, and is found that the unsteady flow at $T a=-50$ is a non-periodic oscillating flow, which oscillates with an irregular pattern that means the flow is chaotic. This chaotic oscillation is well justified by drawing the phase space of the time evolution result as shown in Fig. 8(b). Streamlines and isotherms for the corresponding flow parameters are shown in Fig. 8(c), where it is found that the chaotic oscillation at $T a=-50$ is an asymmetric four- to six-vortex flow patterns. Figure 9(a) shows time evolution of $\lambda$ for $T a=-150$, and it is found that the unsteady flow at $T a=-150$ is a chaotic solutions, which oscillates irregularly with different modes of solutions. This chaotic oscillation is well justified by drawing the phase space of the time evolution result as shown in Fig. 9(b). Typical contours of streamlines and isotherms for $T a=-150$ are shown in Fig. 9(c), where it is found that the chaotic oscillation at $T a=-150$ is an asymmetric four-vortex flow pattern. It is observed that the streamlines of the secondary flow patterns and isotherms of the temperature distributions are significantly distributed that generates more heat which is transferred from the heated wall to the fluid. Then we perform time evolution of $\lambda$ for $T a=-255$ as shown in Fig. 10(a). It is found that the unsteady flow at $T a=-255$ is a periodic oscillation, which is well justified by drawing the phase space as shown in Fig. 10(b). Typical contours streamlines and isotherms for $T a=-255$ are shown in Fig. 10(c), where it is found that the periodic oscillation at $T a=-255$ is an asymmetric four-vortex flow pattern.

(a)

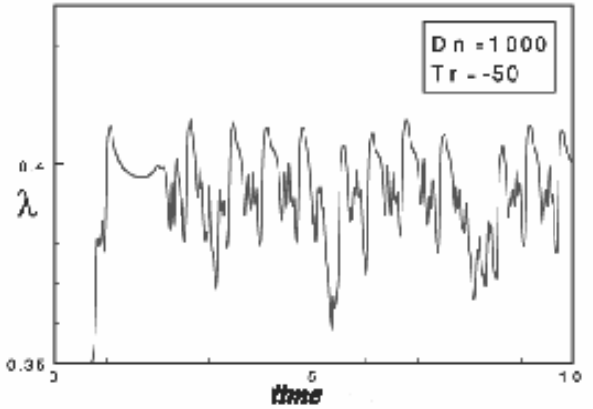

(b)

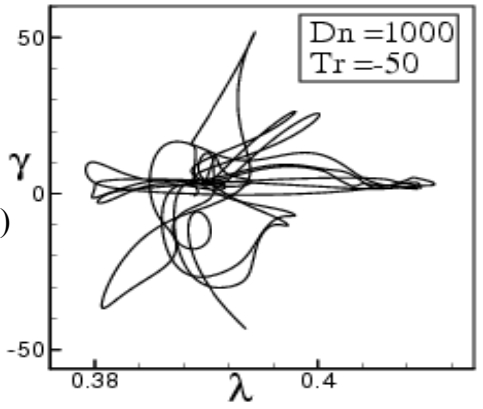




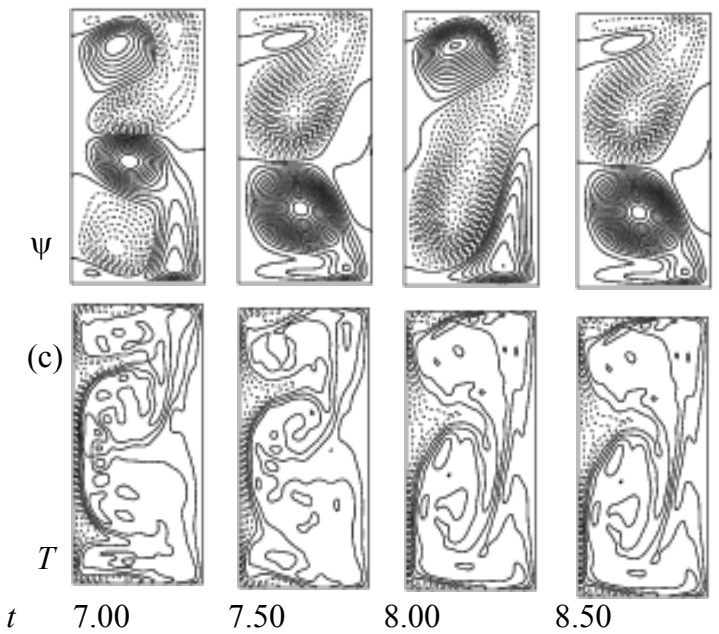

Figure 8. Unsteady results for $T a=-50$. (a) Time evolution of $\lambda$, (b) Phase space, (c) Streamlines (top) and isotherms (bottom) for $7.0 \leq t \leq 8.5$

(a)

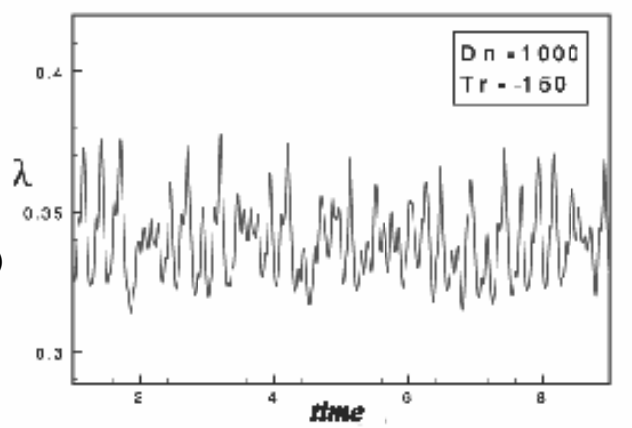

$\psi$

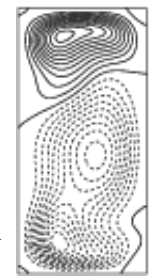

(c)

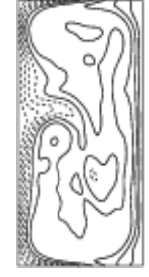

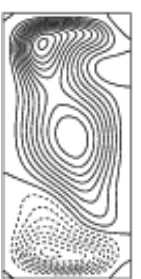

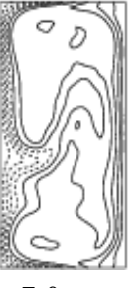

(b)
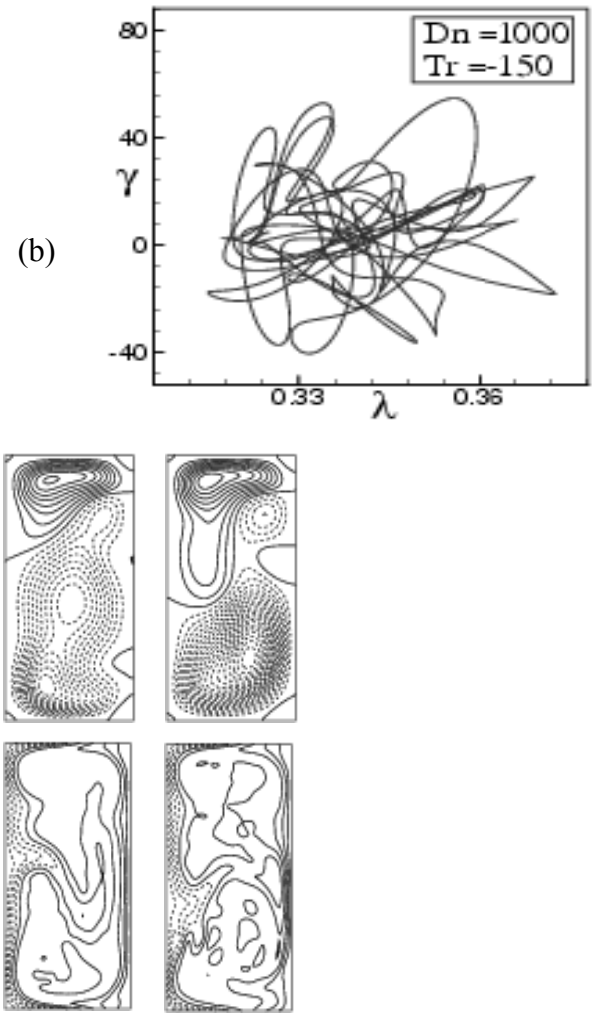

7.5

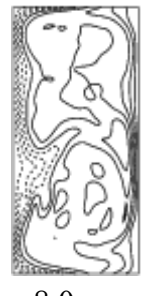

Figure 9. Unsteady results for $T a=-150$. (a) Time evolution of $\lambda$, (b) Phase space, (c) Streamlines (top) and isotherms (bottom) for $6.5 \leq t \leq 8.0$ 

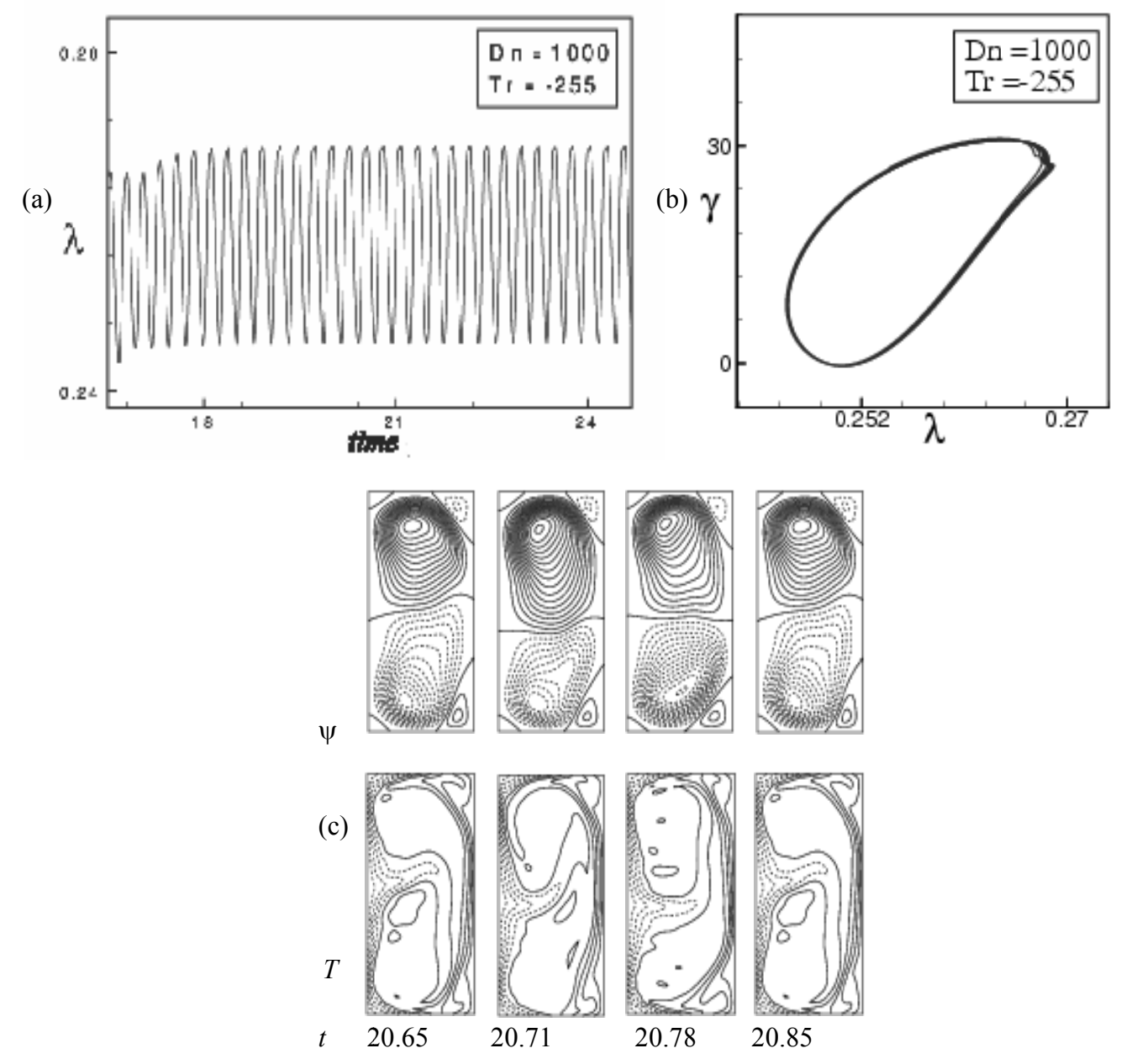

Figure 10. Unsteady results for $T a=-250$. (a) Time evolution of $\lambda$, (b) Phase space, (c) Streamlines (top) and isotherms (bottom) for $20.65 \leq t 20.85$

We then proceeded to perform time evolution of $\lambda$ for $T a=-300$ at $D e=1000$ as shown in Fig. 11(a). As seen in Fig. 11(a), the unsteady flow at $\operatorname{Tr}=-300$ is a time-periodic solution. To observe the time periodic behavior more clearly, we draw the phase space of the time evolution result in Fig. 11(b) for $T a=-300$, where a couple of orbits is observed instead of a single-phase orbit, which confirms that the unsteady flow at $T a=-300$ is a multi-periodic solution. Typical contours of streamlines and isotherms for $T a=-300$ are shown in Fig. 11(c), where it is found that the multi-periodic oscillation at $T a=-300$ is only four-vortex flow pattern. If rotational speed is increased in the negative direction up to $T a=-500$, it is observed that the flow remains steady-state. Figure 12(a) shows, for example, time evolution result for $T a=-320$, where steady-state solution is observed. Therefore, the transition from periodic-oscillating flow to steady-state solution occurs 
between $T a=-300$ and $T a=-320$. Since the flow is steady state, a single contour of the streamlines and isotherms is shown in Fig. 12(b) for $T a=-320$, and it is found that the secondary flow is an asymmetric four-vortex flow pattern. In this study, it is found that if the duct rotation is increased in the positive direction, the number of secondary vortices decreases and consequently heat transfer does not occur substantially; however, for negative rotation, heat transfer is enhanced significantly as the rotation is increased in the negative direction. The reason is that due to chaotic mixing many secondary vortices generate at the outer wall of the duct which play a significant role in transferring heat from the heated wall to the fluid (Chandratilleke et al. [28]).
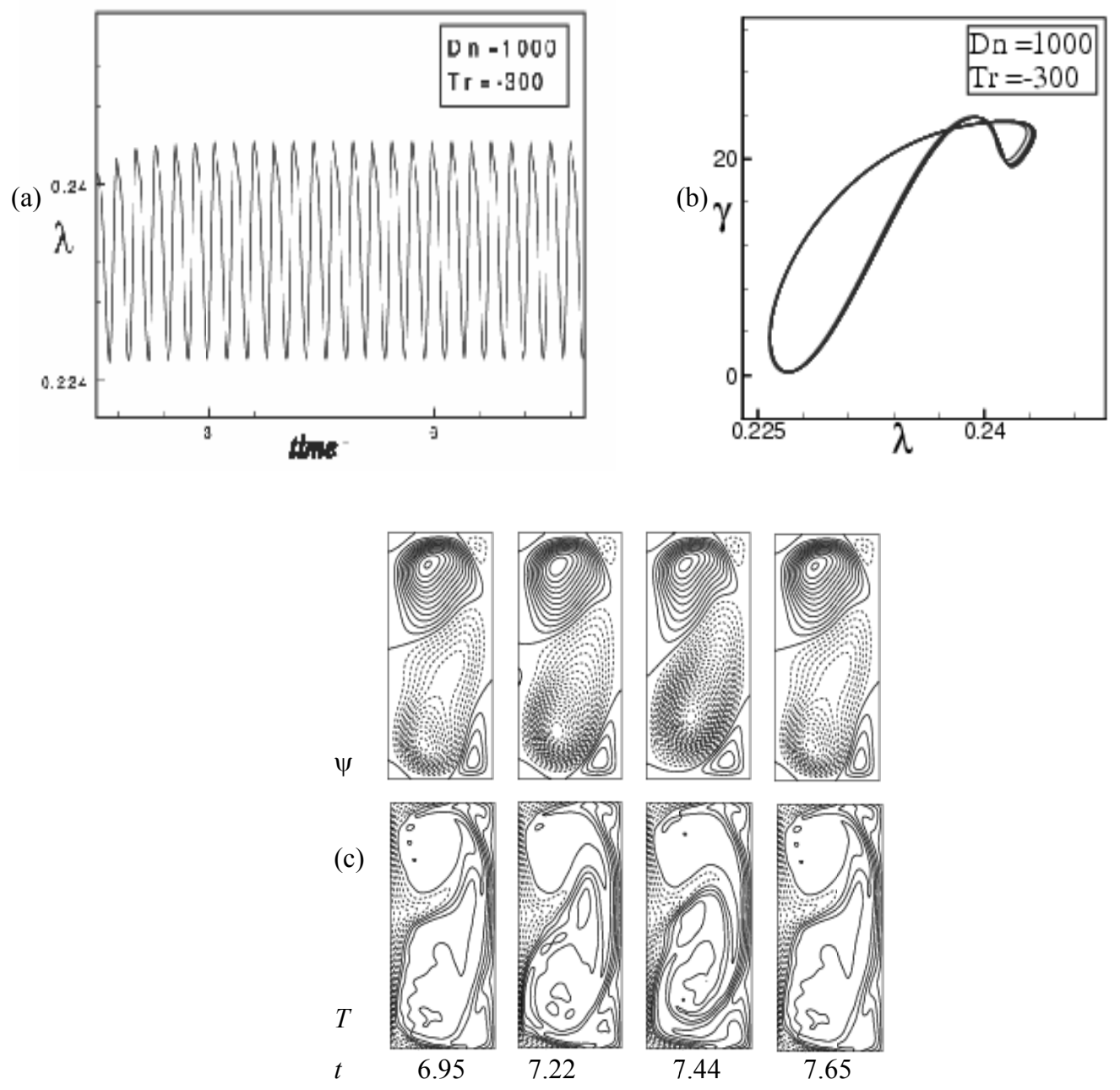

Figure 11. Unsteady results for $T a=-300$. (a) Time evolution of $\lambda$, (b) Phase space, (c) Streamlines (top) and isotherms (bottom) for $6.95 \leq t \leq 7.65$ 


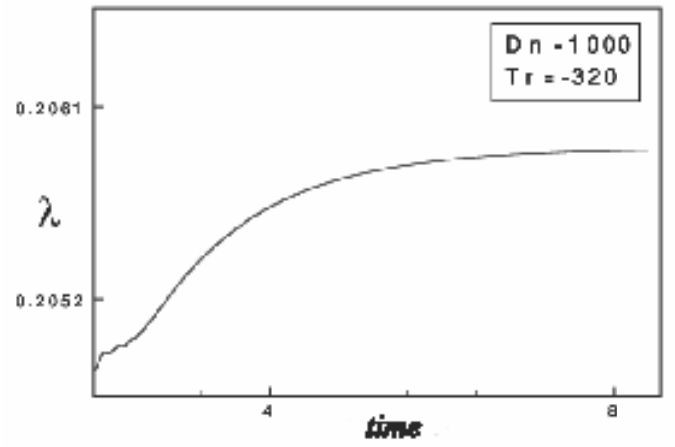

(a)
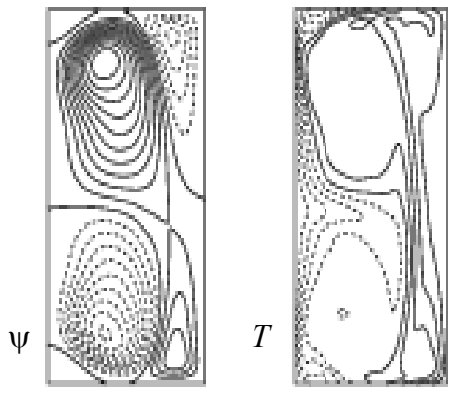

(b)

Figure 12. Unsteady results for $T a=-320$. (a) Time evolution of $\lambda$, (b) Streamlines (top) and isotherms (bottom) at $t=8.0$

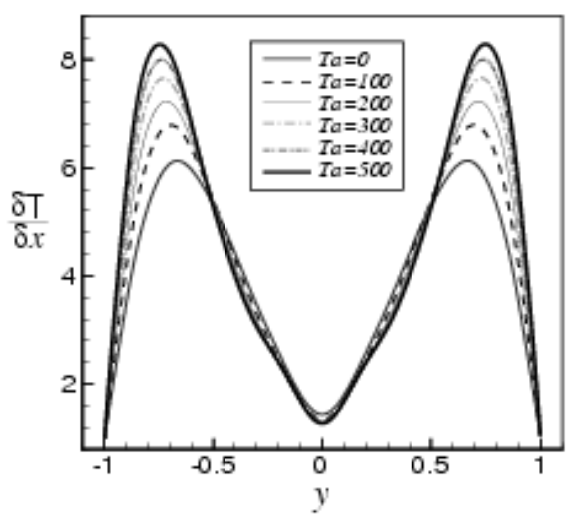

(a)

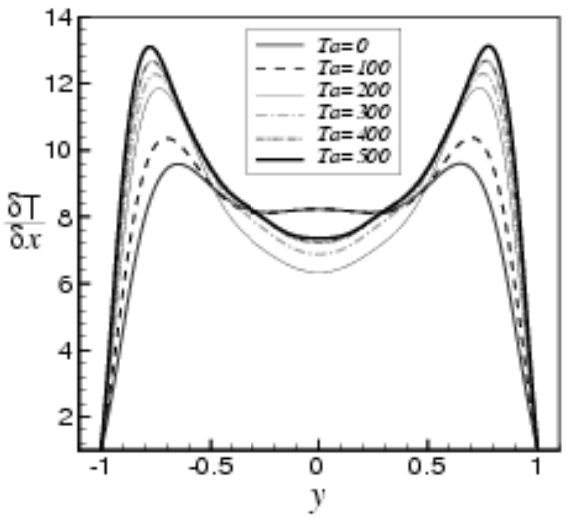

(b)

Figure 13. Temperature gradient for $D e=1000, G r=500$ and $\delta=0.05$ for various vales of $\mathrm{Ta}$. (a) at the cooling wall (b) at the heated wall.

In order to find convective heat transfer from the heated wall to the fluid, we calculate temperature gradients at the cooling and heated walls. In Fig. 13(a), it is shown that $\frac{\partial T}{\partial x}$ on the inner (cooled) sidewall decreases in the central region around $y=0$. This is caused by the advection of the secondary flow in the outward direction around $y=0$ due to the centrifugal force. In the same figure, it is also shown that $\frac{\partial T}{\partial x}$ tends to increase in the regions other than the central region. This is caused by the advection of the secondary flow in the inward direction there, which is a reverse flow of the outward secondary flow in the central region. As seen in Fig. 13(b), on the other hand, the temperature gradient on the outer (heated) sidewall, $\frac{\partial T}{\partial x}$ increases, as the $T a$ goes up, over the whole region except for small $T a$. This is because the secondary flow enhances $\frac{\partial T}{\partial x}$ not 
only in the central region but in other regions as well. This result shows that heat transfer occurs substantially as $T a$ increases for the heated sidewall.

Here we show the validation of our numerical results with the experimental studies. By using visualization method, Yamamoto et al. [29] performed experimental investigations of the flow through a rotating curved square duct of curvature $\delta=0.03$, where three of the duct walls, except the outer wall, rotate around the center of curvature at a constant rotational speed for $T a=150$. In the present study, however, we investigate flow characteristics for the rotation of the whole system (not the three walls only), and show the validation of our study using the same curvature and rotational speed as Yamamoto et al. [29] considered. Figures 14 show comparative study of the experimental vs. numerical results for $T a=150$, and we see that our results have a good agreement with the experimental investigations.

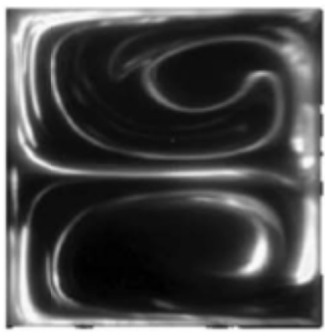

$\mathrm{De}=114$

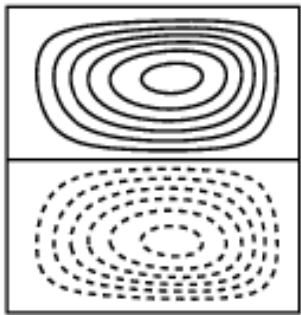

(a)

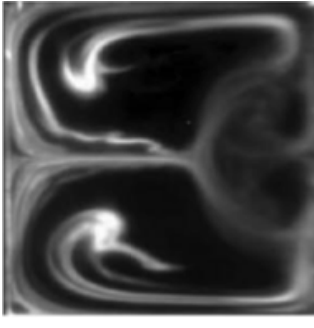

$\mathrm{De}=176$

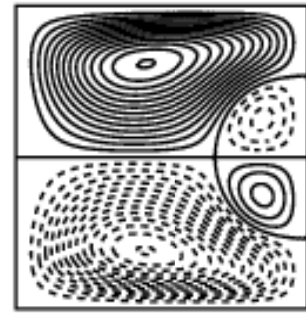

(b)

$\mathrm{De}=415$

Figure 14. Experimental vs. numerical results for rotating curved square duct flow at $T a=150$.

\section{Conclusion}

A spectral-based numerical study is presented for the unsteady flow characteristics through a rotating curved rectangular duct of aspect ratio 2 and curvature ratio 0.05 . Numerical calculations are carried out for the constant Dean number 1000 and Grashof number 500 over a wide range of the Taylor umber $(\mathrm{Ta})$ for both the positive and negative rotation of the duct. The outer wall of the duct is heated while the inner wall cooled, the top and bottom walls being thermally insulated. We investigated unsteady solutions by time evolution calculations justified by their phase spaces, and it is found that under combined action of centrifugal, Coriolis and buoyancy forces the unsteady flow undergoes in the scenario 'chaotic $\rightarrow$ multi-periodic $\rightarrow$ periodic $\rightarrow$ steady-state' if Ta is increased in the positive rotation. For negative rotation, however, time evolution calculations show that the unsteady flow undergoes through various flow instabilities, if $T a$ is increased in the negative direction. Phase spaces were found to be very fruitful to justify the transition of the unsteady flows. Typical contours of secondary flow patterns and temperature profiles are also obtained at several values of $T a$, and it is found that there exist two- to eight-vortex flow patterns if the duct rotation is involved. It is found that the temperature distribution is consistence with the secondary vortices, and secondary flows play a significant role in convective heat transfer from the 
heated wall to the fluid. It is also found that chaotic flow enhances heat transfer more substantially than the steady-state or periodic solutions through multi-vortex flow patterns at the outer concave wall.

\section{REFERENCES}

[1] Dean, W. R., 1927. Note on the motion of fluid in a curved pipe. Philos. Mag., 4: 208-223.

[2] Berger, S.A., Talbot, L. and Yao, L. S., 1983. Flow in Curved Pipes, Annual. Rev. Fluid. Mech., 35: 461-512.

[3] Nandakumar, K and Masliyah, J. H., 1986. Swirling Flow and Heat Transfer in Coiled and Twisted Pipes, Adv. Transport Process., 4: 49-112.

[4] Ito, H, 1987. Flow in Curved Pipes, JSME Int. J., 30: 543-552.

[5] Zhang, J. S. Zhang, B. Z. and Jii J., 2001. Fluid flow in a rotating curved rectangular duct, Int. J. Heat and fluid flow, 22: 583-592.

[6] Ishigaki H., 1996. Laminar Flow in Rotating Curved Pipes. Journal of Fluid Mechanics, 329: 373 388.

[7] Selmi, M., Namdakumar, K. and Finlay W. H., 1994. A bifurcation study of viscous flow through a rotating curved duct, J. Fluid Mech. 262: 353-375.

[8] Dennis, S. C. R. and Ng, M., 1982. Flow in a Curved Channel. Quarterly Journal of Mechanics and Applied Mathematics, 35: 305.

[9] Miyazaki, H., 1973. Combined Free and Force Convection Heat Transfer and Fluid Flow In Rotating Curved Rectangular Tubes. Trans. ASME C: J. Heat Transfer, 95: 64-71.

[10] Selmi, M. and Namdakumar, K., 1999. Bifurcation Study of the Flow Through rotating Curved Ducts, Physics of Fluids, 11: 2030-2043.

[11] Wang, L. Q. and Cheng, K.C., 1996. Flow Transitions and combined Free and Forced Convective Heat Transfer in Rotating Curved Channels: the Case of Positive Rotation, Physics of Fluids, 8: 15531573.

[12] Wang, L. Q. and Cheng, K.C., 1995. Physics Review, E, 51: 1555.

[13] Daskopoulos, P. \& Lenhoff, A. M., 1990. Flow in curved ducts. Part 2. Rotating ducts, Journal of Fluid Mechanics, 217: 575-593.

[14] Yanase, S. and Nishiyama, K., 1988. On the bifurcation of laminar flows through a curved rectangular tube, J. Phys. Soc. Japan, 57(11): 3790-3795.

[15] Yanase, S., Kaga, Y. and Daikai, R., 2002. Laminar flow through a curved rectangular duct over a wide range of the aspect ratio, Fluid Dynamics Research, 31: 151-183.

[16] Wang, L. and Yang, T., 2005. Periodic Oscillation in Curved Duct Flows, Physica D, 200: 296-302.

[17] Mondal, R. N., Alam M. M. and Yanase, S., 2007. Numerical prediction of non-isothermal flows through a rotating curved duct with square cross section, Thommasat Int. J. Sci and Tech., 12: 24-43.

[18] Mondal, R. N., Islam, M. Z. and Islam, M. S., 2013. Transient Heat and Fluid Flow through a Rotating Curved Rectangular Duct: The Case of Positive and Negative Rotation, Procedia Engineering, 56: 179-186.

[19] Mondal, R.N., Ray, S.C. and Yanase S., 2014. Combined Effects of Centrifugal and Coriolis Instability of the Flow through a Rotating Curved Duct with Rectangular Cross Section, Open Journal of Fluid Dynamics, 4: 1-14.

[20] Yamamoto, K., Xiaoyun W., Kazuo N., Yasutaka H., 2006. Visualization of Taylor-Dean Flow in a curved duct of square cross-section, J. Fluid dynamics research. 38: 1-18. 
[21] Nobari, M. R. H., Nousha, A. and Damangir, E., 2009. A Numerical Investigation of Flow and Heat Transfer in Rotating U-Shaped Square Ducts. Int. J. Thermal Sciences, 48: 590-601.

[22] Chandratilleke, T. T. and Nursubyakto, 2003. Numerical prediction of secondary flow and convective heat transfer in externally heated curved rectangular ducts, Int. J. Thermal Sciences, 42(2): 187-198.

[23] Yanase, S., Mondal, R. N. and Kaga, Y., 2005. Numerical Study of Non-isothermal Flow with Convective Heat Transfer in a Curved Rectangular Duct, Int. J. Thermal Sciences, 44: 1047-1060.

[24] Mondal, R. N. Kaga, Y., Hyakutake, T. and Yanase, S., 2006. Effects of curvature and convective heat transfer in curved square duct flows, Trans. ASME, Journal of Fluids Engineering, 128(9): 10131023.

[25] Norouzi, M., Kayhani, M. H., Nobari, M. R. H. and Demneh, M. K., 2009. Convective Heat Transfer of Viscoelastic Flow in a Curved Duct, World Academy of Science, Engineering and Technology, 32: 327-333.

[26] Gottlieb, D. and Orazag, S. A., 1977. Numerical Analysis of Spectral Methods, Society for Industrial and Applied Mathematics, Philadelphia, USA.

[27] Mondal, R. N., Kaga, Y., Hyakutake, T. and Yanase, S., 2007. Bifurcation diagram for twodimensional steady flow and unsteady solutions in a curved square duct, Fluid Dynamics Research, 39: 413-446.

[28] Chandratilleke, T. T., Nadim, N. and Narayanaswamy, R., 2012. Vortex structure-based analysis of laminar flow behavior and thermal characteristics in curved ducts, Int. J. Thermal Sciences, 59: 75-86.

[29] Yamamoto, K., Wu, X., Nozaki, K. and Hayamizu, Y., 2006. Visualization of Taylor-Dean flow in a curved duct of square cross-section, Fluid Dynamics Research, 38(1): 1-18. 